Conclusion* Dynamic dose fractionation HDR-brachytherapy, as 1 st step of preoperative chemo-irradiation, allows to reach the total tumor regression, which enable further ablastic surgery and relapse-free follow-up.

\section{RANDOMISED PHASE II BGOG/ENGOT-CX1 STUDY OF PACLITAXEL-CARBOPLATIN WITH OR WITHOUT NINTEDANIB IN FIRST-LINE RECURRENT OR ADVANCED CERVICAL CANCER}

${ }^{1} \mathrm{BB}$ Vergote ${ }^{*},{ }^{2} \mathrm{E}$ Van Nieuwenhuysen, ${ }^{3} \mathrm{~A}$ Casado, ${ }^{4} \mathrm{~A}$ Laenen, ${ }^{5} \mathrm{D}$ Lorusso, ${ }^{6} \mathrm{~B}$ Braicu, ${ }^{7} \mathrm{E}$ Guerra-Alia, ${ }^{8} \mathrm{P}$ Zola, ${ }^{9} \mathrm{P}$ Wimberger, ${ }^{10} \mathrm{P}$ Debruyne, ${ }^{11} \mathrm{E}$ Falcó, ${ }^{12} \mathrm{~A}$ Ferrero, ${ }^{13} \mathrm{MZ}$ Muallem, ${ }^{14} \mathrm{~J}$ Kerger, ${ }^{15} \mathrm{E}$ Garcia Martinez, ${ }^{16} \mathrm{~S}$ Pignata, ${ }^{17 J} \mathrm{~J}$ Sehoulli, ${ }^{2} \mathrm{~T}$ Van Gorp, ${ }^{18} \mathrm{C}$ Gennigens, ${ }^{19} \mathrm{MJ}$ Rubio Pérez. ${ }^{1}$ University Hospitals Leuven, Belgium and $B G O G$, Belgium; ${ }^{2}$ University Hospitals Leuven, Belgium and BGOG; ${ }^{3}$ Hospital Clínico San Carlos, Madrid Spain and GEICO; ${ }^{4}$ Catholic University Leuven, Belgium; ${ }^{5}$ Policlinico Universitario Agostino Gemelli, Italy and ENGOT; ${ }^{6}$ Charité Universitätsmedizin Berlin and ENGOT; ${ }^{7}$ Hospital Ramón y Cajal, Madrid Spain and GEICO; ${ }^{8}$ Univeristy of Turin, Italy and ENGOT; ${ }^{9}$ Universitätsklinikum Carl Gustav Carus, Dresden, Germany and ENGOT; ${ }^{10}$ AZ Groeninge, Kortrijk, Belgium; ${ }^{11}$ Hospital Son Llatzer, Palma de Mallorca, Spain and GEICO ${ }^{12}$ Mauriziano Hospital, Torino, Italy and ENGOT; ${ }^{13}$ Charité Universitätsmedizin Berlin, Berlin, Germany; ${ }^{14}$ Institut Jules Bordet, Brussels, Belgium and BGOG; ${ }^{15}$ Hospital Universitario Morales Meseguer, Murcia Spain and GEICO; ${ }^{16}$ Instituto Nazionale Tumor, Napoli, Italy and ENGOT; ${ }^{17}$ Charité Universitätsmedizin Berlin, Berlin, Germany and ENGOT; ${ }^{18}$ University Hospital (CHU) of Liege, Belgium and BGOG; ${ }^{19}$ Hospital Universitario Reina Sofía, Córdoba, Spain and GEICO

\subsection{6/ijgc-2021-ESGO.16}

Introduction/Background* Platinum in combination with paclitaxel $(\mathrm{P})$ and bevacizumab is the standard of care in first-line recurrent/advanced cervical cancer (Tewari, NEJM 2020). Nintedanib is an oral tyrosine kinase inhibitor targeting, among others, vascular endothelial growth factor receptor.

Methodology Double-blind phase II randomised study in patients with first-line recurrent or primary advanced (FIGO stage IVB) cervical cancer. Patients received carboplatin AUC 5-6 and paclitaxel $175 \mathrm{mg} / \mathrm{m}^{2}$ q 3 weeks with oral nintedanib $200 \mathrm{mg} \mathrm{BID} /$ placebo. Stratification factor was primary advanced versus recurrent disease. The primary endpoint was progression-free survival (PFS) at 1,5 years with at least 87 events and $\alpha=0.15, \beta=80 \%$, one sided, in favor of the nintedanib (N) versus control (C) arm. The study (NCT02009579) was performed according to the ENGOT model A.

Result(s)* 120 patients (62 N, 58 C) were randomised between March 2014 and October 2018. Median follow-up was 35 months. Baseline characteristics were similar in both groups (total population: squamous cell carcinoma 62\%, prior radiotherapy $64 \%$, primary advanced $25 \%$, recurrent $75 \%$ ). The primary endpoint was met with a PFS at 1.5 years of $15.1 \%$ versus $12.8 \%$ in favour of the nintedanib arm $(\mathrm{p}=$ 0.057). Median overall survival (OS) was 21.7 and 16.4 months for $\mathrm{N}$ and $\mathrm{C}$, respectively. Subgroup analysis did not demonstrate a difference in PFS in the primary advanced setting, but in the recurrent setting the 1 year PFS was $22.8 \%$ and $14.9 \%$ for $\mathrm{N}$ and $\mathrm{C}$, respectively. Confirmed RECIST response rate was $48 \%$ for $\mathrm{N}$ and $39 \%$ for $\mathrm{C}$. No new adverse events were noted for $\mathrm{N}$. However $\mathrm{N}$ was associated with numerically more serious adverse events for anemia and febrile neutropenia. Discontinuation of chemotherapy was similar in both groups. $\mathrm{N}$ was discontinued in $3 \%$ versus placebo in $1.6 \%$ of the patients. Dose reduction of $\mathrm{N}$ was necessary in $53 \%$ of the patients.
Conclusion* The study met its primary endpoint with a prolonged PFS in the $\mathrm{N}$ arm. No new safety signals were observed.

\section{CAN METHYLATION SIGNALS OF CERVICAL CANCER ENHANCE CERVICAL SCREENING?: AN EPIGENOME- WIDE ASSOCIATION STUDY ON THE ILLUMINA 850K ARRAY}

\begin{abstract}
1;2S Bowden ${ }^{*},{ }^{3} \mathrm{~B}$ Bodinier, ${ }^{4} \mathrm{M}$ Paraskevaidi, ${ }^{1}$ Kalliala, ${ }^{5} \mathrm{~N}$ Chaitrakulthong, ${ }^{1} \mathrm{~A}$ Mitra, ${ }^{1} \mathrm{M}$ Tzafetas, ${ }^{4} \mathrm{~K}$ Lathouras, ${ }^{6} \mathrm{M}$ Nasioutziki, ${ }^{5}$ J Flanagan, ${ }^{3} \mathrm{M}$ Chadeau-Hyam, ${ }^{2 ; 4} \mathrm{M}$ Kyrgiou. ${ }^{1}$ Instiute of Reproductive and Developmental Biology, Department of Surgery and Cancer Metabolism, Digestion and Reproduction, UKi ${ }^{2}$ West London Gynaecological Cancer Centre, Hammersmith Hospital, London, UK; ${ }^{3}$ Imperial College London, School of Public Health , Biostastistics and Epidemiology, London; ${ }^{4}$ Imperial College London, Institute of Reproductive and Developmental Biology, Department of Surgery and Cancer - Metabolism, Digestion and Reproduction, UK; ${ }^{5}$ mperial College London, Institute of Developmental and Reproductive Biology, Surgery and Cancer, London, UK; ${ }^{6}$ Aristotle University, Cytopathology, Thessaloniki, Greece
\end{abstract}

\subsection{6/ijgc-2021-ESG0.17}

Introduction/Background* Cervical cancer is one of the most prevalent and leading cause of cancer mortality for women worldwide. Persistent infection with HPV is associated with the development of cervical cancer. Although most infections are cleared by the immune response, the factors related to persistent infection and carcinogenesis are less well understood. Epigenetic alterations are essential in the development of many cancers; while aberrant hypermethylation in cervical carcinogenesis has been identified in several targeted studies, epigenome-wide exploration has been limited. In this epigenome-wide association study (EWAS) we explore differential DNA methylation signatures associated to CIN3 and Cervical Cancer, to better understand potential drivers and biomarkers of cervical carcinogenesis.

Methodology 247 women (119 normal, 74 CIN3/CGIN and 54 cervical cancer) attending gynaecological appointments, cervical screening and oncological treatment between 2014-2020 at English and Greek referral hospitals were recruited. Methylation signatures were obtained following bisulphite conversion of DNA extracted from exfoliated cervical cells and sequenced using the Illumina $850 \mathrm{k}$ array data. After normalisaton and data QC, mixed linear models and a penalised regression were used to test for independent associations between methylation $\mathrm{CpG}$ sites and case-control status. P-values were Bonferroni corrected and adjusted for batch, chip, age and HPV status.

Result(s)* $177 \mathrm{CpG}$ sites were strongly assosicated with CIN3 or cervical cancer, as measured by a $P$-value $<5 \times 10^{-8}$ and a gain/loss in methylation of $>15 \%$ between cases and controls. Epigenetic signals were located in over 141 unique genes genome-wide, with a gain in methylation in CIN3 or cancer cases being more common $(n=77 / 80)$.

Conclusion* This is potentially the first adequately powered study to highlight genome-wide epigenetic signatures associated to carcinoma in situ and cervical cancer. This builds on previous genetic signals associated with cervical cancer identified by our group (Bowden et al, Lancet Oncol, 2021). While around 59 genes were previously reported associations to CIN or cervical cancer, the remaining 82 signals appear to be novel. Functional annotation highlighted several genes related to tumour suppresor function, transcription factors and apoptosis - important for cervical cancer tumorigenesis. 